\title{
Knowledge-Aided Multichannel Adaptive SAR/GMTI Processing: Algorithm and Experimental Results
}

\author{
Di Wu, Daiyin Zhu, and Zhaoda Zhu \\ College of Information Science and Technology, Nanjing University of Aeronautics \& Astronautics, Nanjing 210016, China \\ Correspondence should be addressed to Di Wu, wudi_nuaa@yahoo.cn
}

Received 30 May 2009; Revised 5 November 2009; Accepted 18 January 2010

Academic Editor: Andreas Reigber

Copyright () 2010 Di Wu et al. This is an open access article distributed under the Creative Commons Attribution License, which permits unrestricted use, distribution, and reproduction in any medium, provided the original work is properly cited.

The multichannel synthetic aperture radar ground moving target indication (SAR/GMTI) technique is a simplified implementation of space-time adaptive processing (STAP), which has been proved to be feasible in the past decades. However, its detection performance will be degraded in heterogeneous environments due to the rapidly varying clutter characteristics. Knowledge-aided (KA) STAP provides an effective way to deal with the nonstationary problem in real-world clutter environment. Based on the KA STAP methods, this paper proposes a KA algorithm for adaptive SAR/GMTI processing in heterogeneous environments. It reduces sample support by its fast convergence properties and shows robust to non-stationary clutter distribution relative to the traditional adaptive SAR/GMTI scheme. Experimental clutter suppression results are employed to verify the virtue of this algorithm.

\section{Introduction}

Synthetic aperture radar (SAR) is an important sensor that can reconstruct the reflectivity image of the ground stationary scene. Ground moving target indication (GMTI) with SAR has been widely explored for both military and civilian task. Moving target detection in SAR image is a difficulty, because the slowly moving target may be totally submerged among the main-beam clutter in spatial, time, and frequency domain. Space-time adaptive processing (STAP) [1-3] is a leading technology candidate for improving detection performance of airborne and spaceborne radar in strong clutter and interference environments. A simple, but of most practical importance, SAR/STAP scheme is the wellknown multichannel along-track interferometric (ATI) SAR which has been widely used in the past decades $[4,5]$. It utilizes two or more receiving channels whose baseline lies along the flight direction, SAR images from different channels concerning the same scene are generated, and clutter suppression can be achieved by combining these complex images. Actually, the "combining" mentioned above can be seen as a beamforming process in image domain and the weights should be estimated adaptively from the secondary data due to the unknown characteristics of the interference (considered to be composed of clutter and thermal noise in this paper).

It has been indicated in many literatures (e.g., [68]) that STAP performance can be severely degraded in heterogeneous environments. As a simplified STAP, adaptive SAR/GMTI system is confronted with the same problem. Since the adaptive weights depend on two unknown quantities, that is, the clutter-plus-noise $(C+N)$ covariance matrix and the target steering vector, the precise estimation of the covariance matrix in heterogeneous environments presents to be a key for the detection tasks.

Knowledge-aided (KA) STAP such as colored loading and data prewhitening techniques has been proposed to mitigate the performance loss due to realistic environments with fast varying clutter characteristics [9-12]. It incorporates a priori knowledge in the traditional local training schemes (estimate the $C+N$ covariance matrix concerning the pixel under test by averaging over the adjacent range pixels) to obtain a more precise estimation of the characteristics of interference. Everything from digital terrain, elevation data, SAR images, even hyperspectral imagery can be exploited as a priori knowledge. It is intuitive to believe that the same KA scheme can also be introduced to the adaptive SAR/GMTI processing in heterogeneous environments. 
In this paper, we focus our research on the use of a priori knowledge in SAR/GMTI detection processing. A parametric model of multichannel SAR $C+N$ covariance matrix is investigated at first, which can be employed as the prior form to be incorporated into the local training scheme. Based on that, a KA adaptive SAR/GMTI algorithm is proposed and verified by experimental results to be effective in real-world clutter environment.

The remainder of this paper is organized as follows. Section 2 briefly reviews the fundamental of a multichannel SAR detection processing and analyzes the impact of heterogeneous environments. In Section 3, a priori model of the $C+N$ covariance matrix is formulated, and a KA adaptive SAR/GMTI algorithm is proposed to operate in the real-world clutter environments. In Section 4, experimental clutter suppression results are provided to demonstrate the desirable improvement of the proposed KA method. At last, Section 5 provides a summary and conclusions. All the experimental results shown in this paper are obtained from the measured data collected by a three-channel ATI SAR system.

Notation. Vectors are denoted by boldface lowercase letters and matrices by boldface uppercase letters. We use superscripts $T,{ }^{*}$, and $H$, to denote the transpose, conjugate and transpose conjugate operation, respectively. The Frobenius matrix norm is denoted as $\|\cdot\|$ and the expectation operator as $E\{\cdot\}$.

\section{Multichannel SAR/GMTI in Heterogeneous Environments}

This section develops a brief review of moving target detection flaw for a multichannel SAR system. The optimal beamforming weight vector maximizing the output signalto-interference-plus-noise ratio (SINR) and the impact of a non-stationary clutter distribution are investigated. We validate the performance loss due to heterogeneous environments using measured data taken from a three-channel SAR system.

\subsection{Multichannel SAR/GMTI Detection Architecture. A mul-} tichannel SAR/GMTI detection processing scheme is shown in Figure 1. SAR processing at each receiving channel is followed by a modified $\Sigma$ map formation via applying a pixel-by-pixel beamforming technique between the complex maps from different channels. By judicious setting of the beamforming weight vector, the clutter component can be filtered out, leaving only the desired targets. CFAR processing over the $\Sigma$ map enables the detection of moving targets.

Consider a multichannel SAR system with $K$ receiving channels; the returns from each transmitted pulse are received by all the channels and undergo SAR processing to produce $K$ images. As we know, Many successful SAR imaging algorithms have been widely used [13], for example, the range-Doppler (RD), chirp scaling (CS), and omega$\mathrm{k}$ algorithm, and so forth. In this paper, the polar format algorithm (PFA) $[14,15]$ is utilized for the images generation. Thus, for a certain image pixel, there are $K$ complex samples from different channels, which are represented by $x_{1}, x_{2}, \ldots, x_{K}$. The beamformer input and weight vector can be represented by the $K \times 1$ column vectors $\mathbf{x}=$ $\left[x_{1}, x_{2}, \ldots x_{K}\right]^{T}$ and $\mathbf{w}=\left[w_{1}, w_{2}, \ldots, w_{K}\right]^{T}$, respectively. The well-known optimum weight vector maximizing the output SINR is [16]

$$
\mathbf{w}_{\mathrm{opt}}=\gamma \mathbf{R}^{-1} \mathbf{s},
$$

where $\gamma$ is an arbitrary constant, and $\mathbf{R}$ and $\mathbf{s}$ are the clutterplus-noise $(C+N)$ covariance matrix and desired signal steering vector, respectively. In practice, due to the unknown statistics of the interference environment, the covariance matrix associated with a certain pixel is never known a priori. Thus for adaptive processing, it is common to replace $\mathbf{R}$ with its maximum likelihood estimation (MLE) [1-3]:

$$
\widehat{\mathbf{R}}=\frac{1}{L} \sum_{i=1}^{L} \mathbf{x}(i) \mathbf{x}^{H}(i) .
$$

In (2), $\mathbf{x}(i)$ is the sample vector from the range pixel close to the pixel under test which is also called the secondary data. An important assumption for (2) is that the samples are drawn from an independent and identically distributed (i.i.d) Gaussian stochastic process. Substituting (2) into (1) yields the weight vector

$$
\widehat{\mathbf{w}}=\gamma \hat{\mathbf{R}}^{-1} \mathbf{s} .
$$

The literature commonly refers to the implementation in (3) as sample matrix inversion (SMI). With this weight vector, the beamformer's output can be represented as

$$
x_{\Sigma}=\widehat{\mathbf{w}}^{H} \mathbf{x}
$$

After applying the aforementioned steps for every pixel, the weighted $\Sigma$ map mentioned above is formed for the next CFAR processing.

2.2. Performance Loss in Heterogeneous Environments. From (3) we note that since the inverted clutter-plus-noise covariance matrix is employed for weight training, the ability to accurately estimate $\mathbf{R}$ is decisive to a successful detection. It is clear that real-world ground clutter model will not always observe the i.i.d assumption. Variations in the underlying terrain, the intrinsic clutter motion, and the target-like signals will contribute to stationary violations [6-10]. In SAR system with high resolution, clutter properties change rapidly over image pixels. Such effects lead to covariance matrix estimation errors, adaptive filter mismatch, and consequently, performance loss in GMTI processing.

To demonstrate the aforementioned phenomena, several experimental results obtained from the three-channel SAR system with relevant parameters listed in Table 1 are shown herein. Figure 2 captures the estimated SINR loss [1] concerning a certain pixel under test when training over $6,12,24$ samples derived from the adjacent range pixels. 


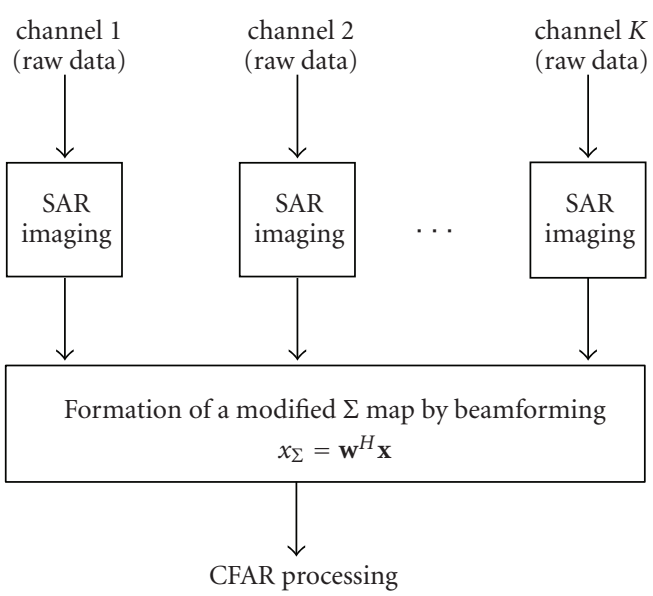

(a) Detection processing flaw

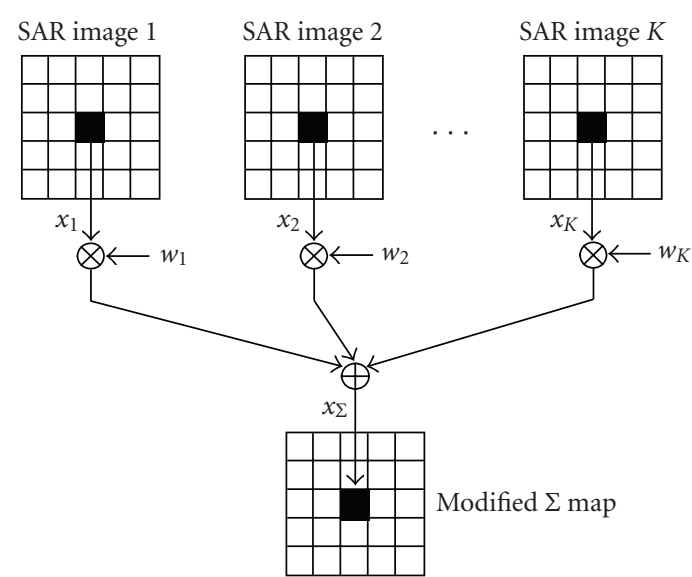

(b) Element-by-element beamforming

FIGURE 1: Multichannel SAR/GMTI detection Scheme.

TABLE 1: Parameters of the three-channel ATI SAR system.

\begin{tabular}{lc}
\hline Parameter & Value \\
\hline center frequency & X band \\
antenna length & $1 \mathrm{~m}$ \\
bandwidth & $200 \mathrm{MHz}$ \\
PRF & $1250 \mathrm{~Hz}$ \\
number of pulses & 1024 \\
distance between each antenna & $0.33 \mathrm{~m}$ \\
port phase center & $110 \mathrm{~m} / \mathrm{s}$ \\
platform speed & $5.0 \mathrm{~km}$ \\
platform altitude & $24 \mathrm{~km}$ \\
distance between antenna phase & \\
center and scene center &
\end{tabular}

Since we can never know exactly the true $C+N$ covariance matrix in practice, the SINR loss curve training over 64 samples with high homogeneity was superimposed here as an approximation of the real case. Note the inaccurate notch's width, nulling placement, and insufficient gains at normalized Doppler approaching $-0.2 \sim-0.3$ for the estimated curves. Actually, the false model is a result of fast varying clutter distribution and target-like signals corrupting the secondary data. As we know, unfaithful model of SINR loss curve is tantamount to the clutter residue, target cancellation, and increasing minimum detectable velocity (MDV), which finally leads to poor detection ability of a GMTI system.

Given the impact of non-stationary clutter environments above, the traditional adaptive SAR/GMTI scheme based on the covariance matrix estimation in (2) is prone to be erroneous in heterogeneous clutter environments. It is inappropriate to consider a covariance matrix estimation method simply using (2). In the next section, a priori knowl-

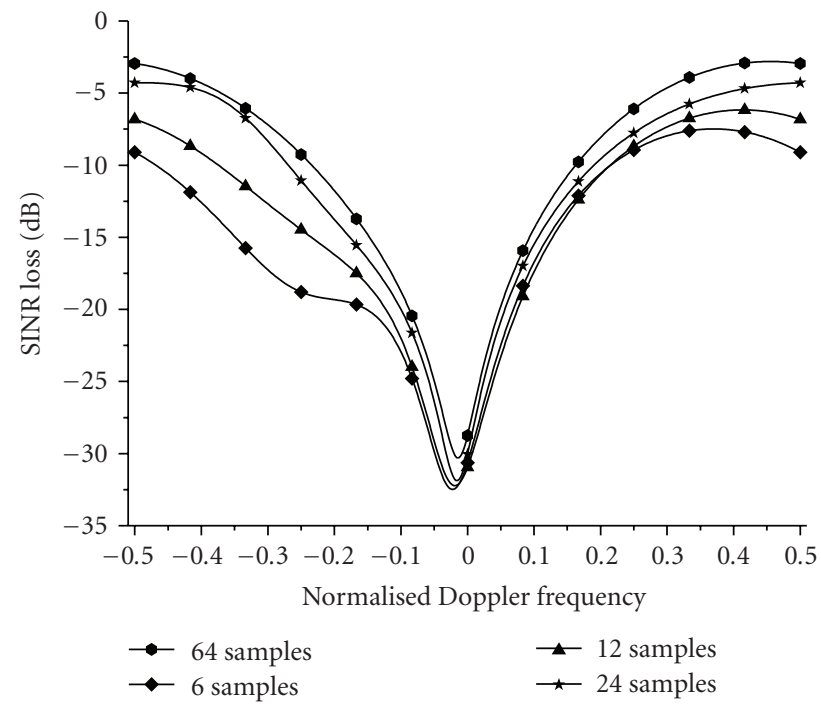

FIGURE 2: Estimated SINR loss curve with different number of training samples.

edge is introduce to construct the weight vectors, and the knowledge-aided adaptive SAR/GMTI algorithm is proposed to enhance the detection performance in heterogeneous environments.

\section{Knowledge-Aided Adaptive SAR/GMTI}

Knowledge-aided STAP such as colored loading and data prewhitening techniques has been proposed to enhance detection performance in heterogeneous environment [912]. It incorporates an initial guess of $\mathbf{R}$, let us say $\mathbf{R}_{0}$, formed via a priori knowledge in the estimation of $\mathbf{R}$. According to the theory of Bayesian estimation, the improved estimate $\widetilde{\mathbf{R}}$ 


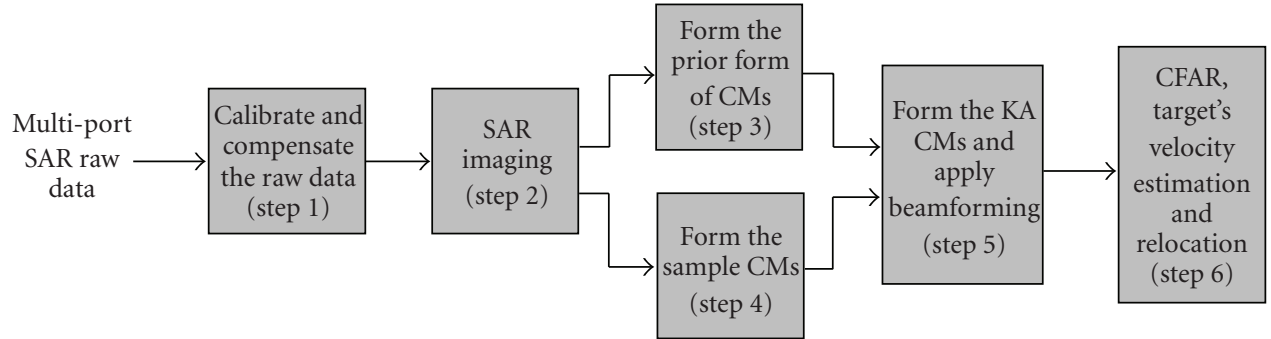

Figure 3: Knowledge-aided adaptive SAR/GMTI flow diagram.

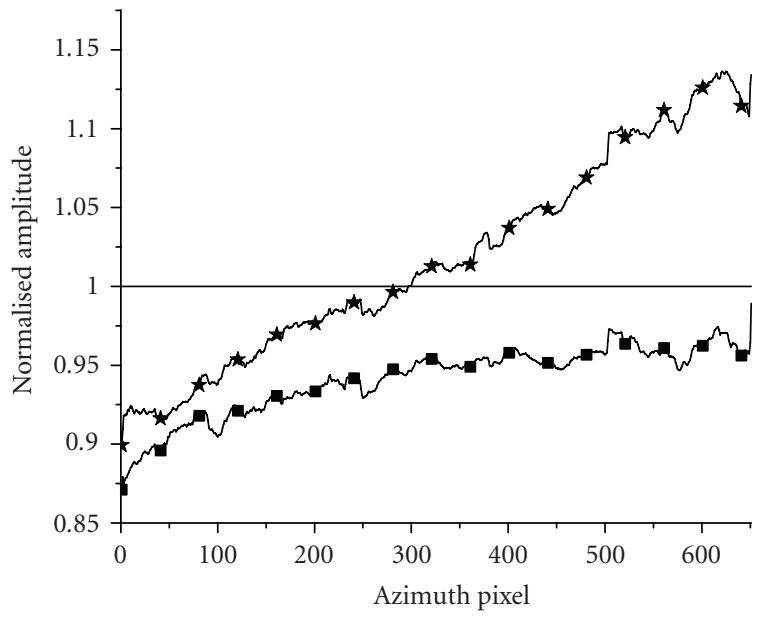

(a) Amplitude responses before step1

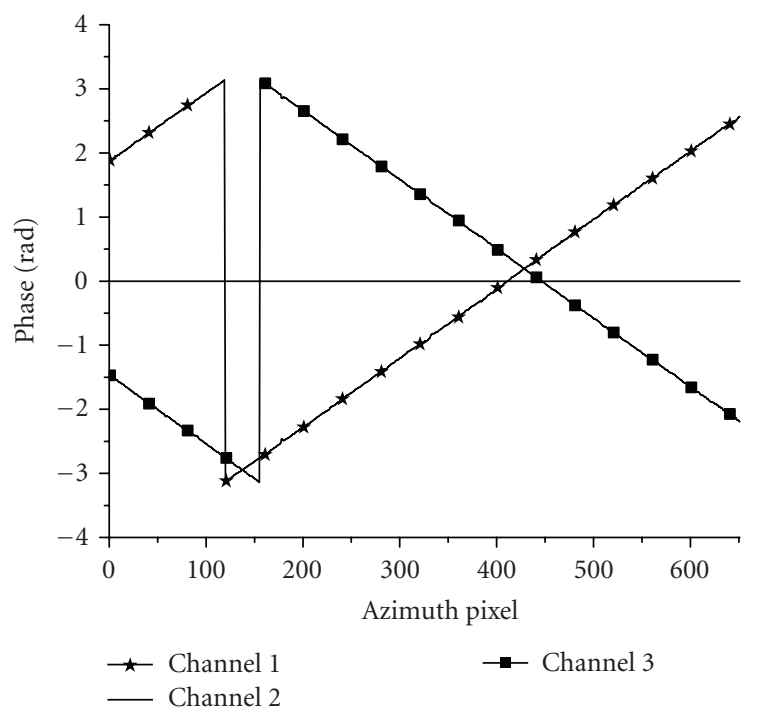

(c) Phase responses before step1

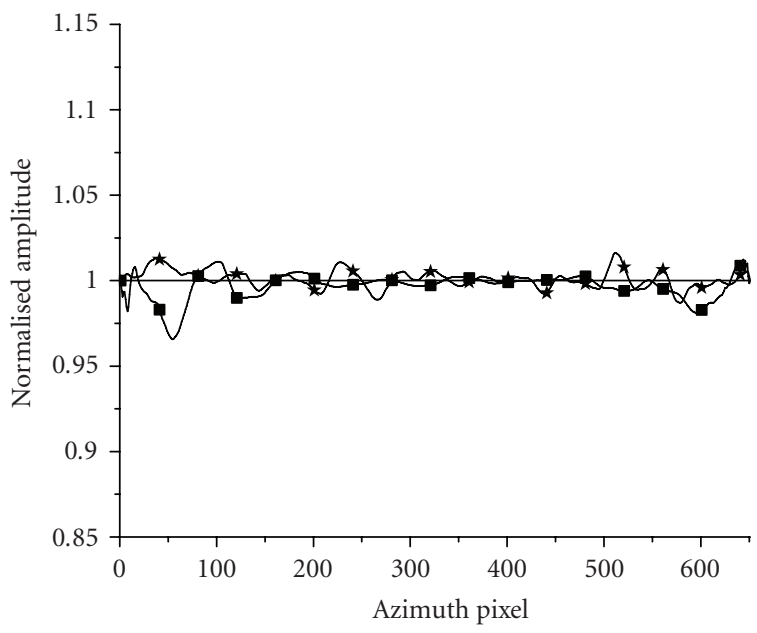

(b) Amplitude responses after step1

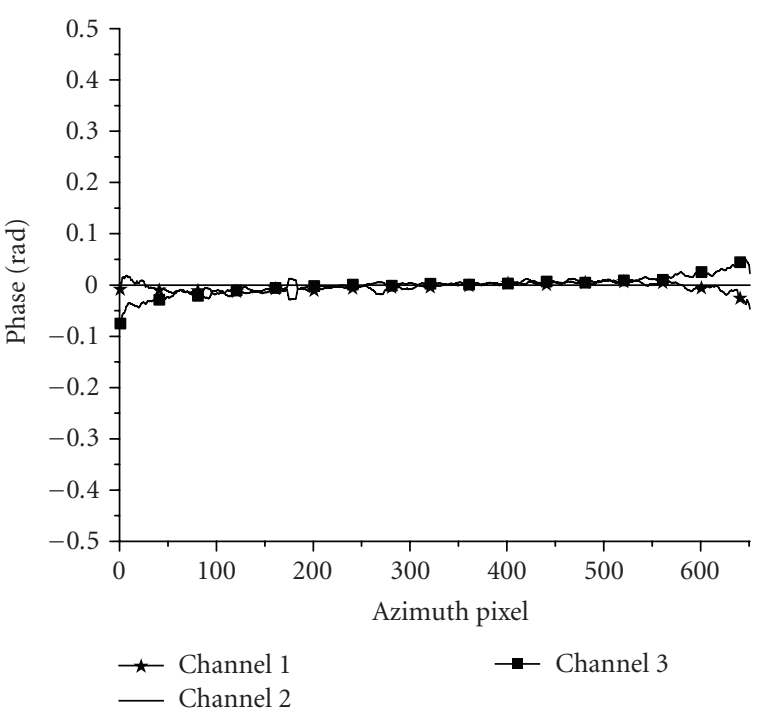

(d) Phase responses after step1

FIGURE 4: Array responses before and after step1.

is given by a linear combination of $\mathbf{R}_{0}$ and $\hat{\mathbf{R}}$ [17]:

$$
\widetilde{\mathbf{R}}=\alpha \mathbf{R}_{0}+(1-\alpha) \hat{\mathbf{R}} ; \quad \alpha \in(0,1) .
$$

A method to choose $\alpha$ adaptively so as to maximally whiten the observed interference data is provided in [9]. Recently, new approaches for selecting $\alpha$ have been proposed in $[11,12]$, which enable us to obtain an automatic estimate of $\alpha$ directly from the received data set via the principle of minimum mean-squared error (MSE).

Based on these KA STAP techniques, a KA adaptive SAR/GMTI algorithm for multichannel SAR system is proposed in the following discussion, which can be considered 


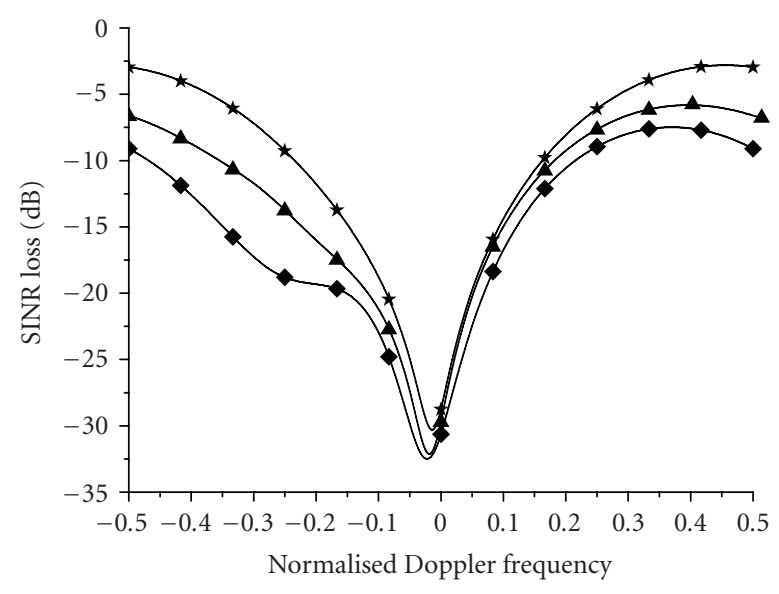

(a) 6 samples used

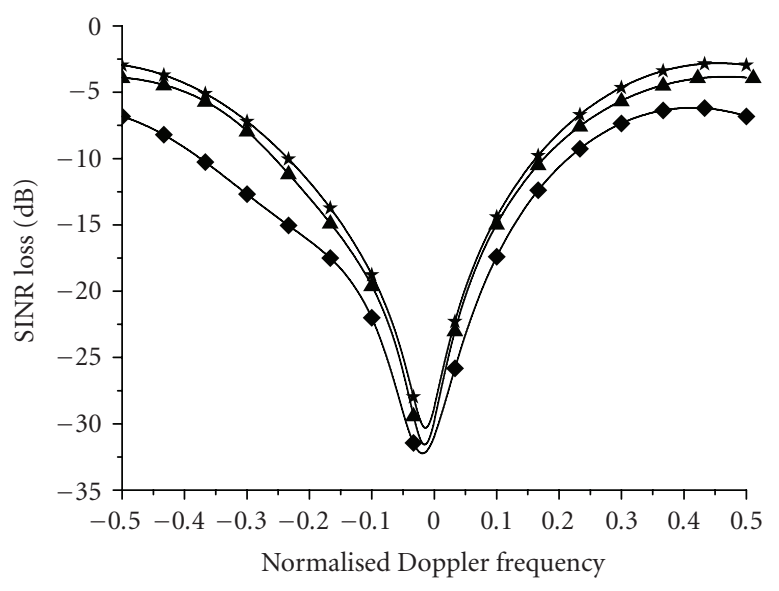

(b) 12 samples used

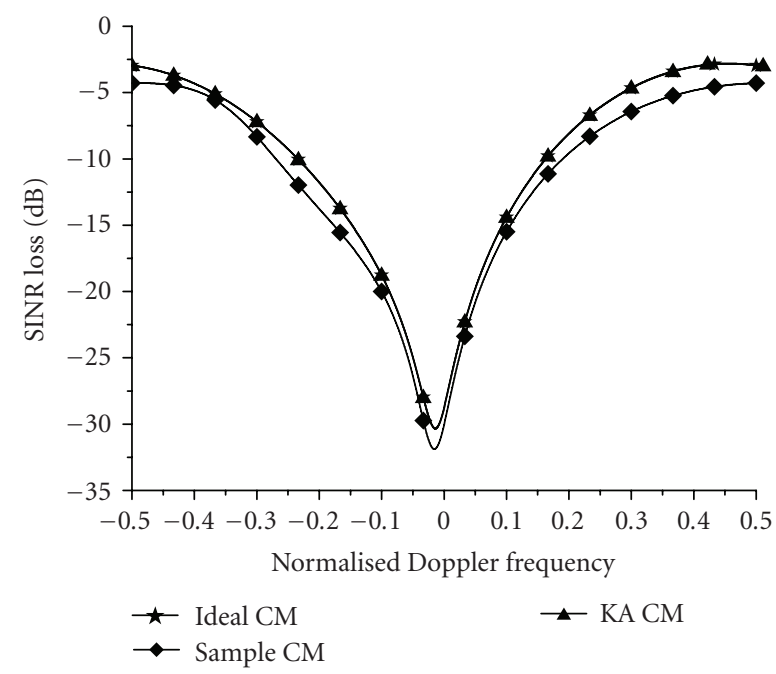

(c) 24 samples used

FIGURE 5: Comparison of SINR loss curves for local training and KA method.

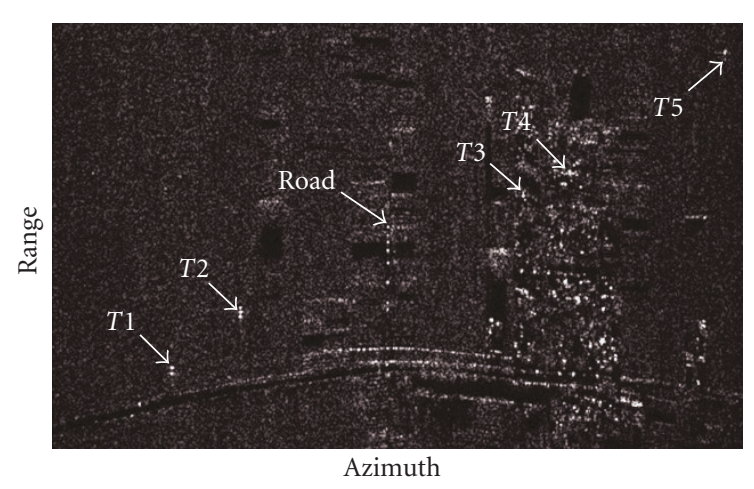

FIgURE 6: SAR image concerning the detection area.

as an extension of the KA STAP schemes. However, it is in possession of many particular characteristics and can be conveniently implemented in practical SAR/GMTI processing.
3.1. A Priori Form of the Clutter-Plus-Noise Covariance Matrix. As the clutter-plus-noise covariance matrix is central to adaptive beamforming, we begin by building up a priori form of the multichannel SAR $C+N$ covariance matrix that helps motivate the KA SAR/GMTI algorithm.

Because of the two-dimensional high resolution, interference present at a certain image pixel can be seen as clutter echo received from a single clutter patch plus a sampled value of thermal noise. The interference vector received on the multichannel SAR system with $K$ receiving elements can be represented by the $K \times 1$ vector as

$$
\mathbf{x}_{I}=\alpha_{c} \mathbf{c}(\theta, \varphi) \odot \boldsymbol{\varepsilon}(\theta, \varphi) \odot\left(\mathbf{1}_{K \times 1}+\mathbf{t}_{p}(\theta, \varphi)\right)+\mathbf{n},
$$

where $\alpha_{c}, \theta, \varphi$ are the complex amplitude, elevation, and azimuth angle concerning the clutter from a certain patch, $\odot$ is the Hadamard product, $\mathbf{c}(\theta, \varphi)$ denotes the ideal clutter spatial steering vector without any modulation, $\boldsymbol{\varepsilon}(\theta, \varphi)$ is the angle dependent array error vector, and $\mathbf{n}$ represents the vector of sampled thermal noise. $\mathbf{1}_{K \times 1}+\mathbf{t}_{p}(\theta, \varphi)$ in the parenthesis represents small unknown random modulations 


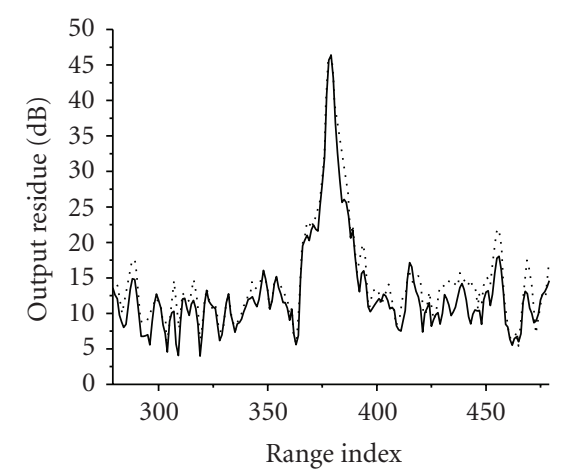

(a) target 1

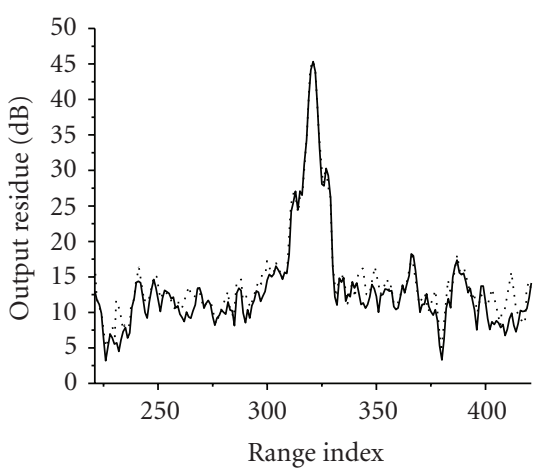

(b) target 2

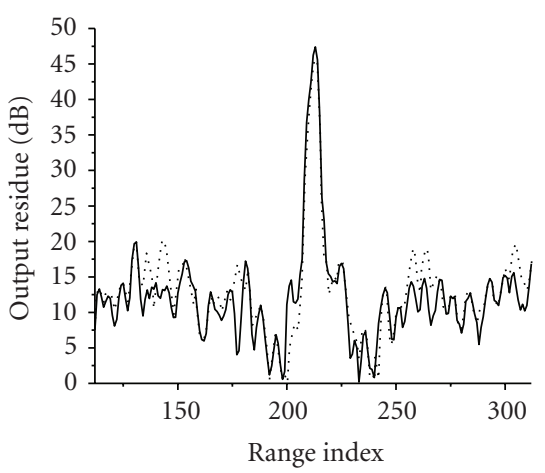

(c) target 3

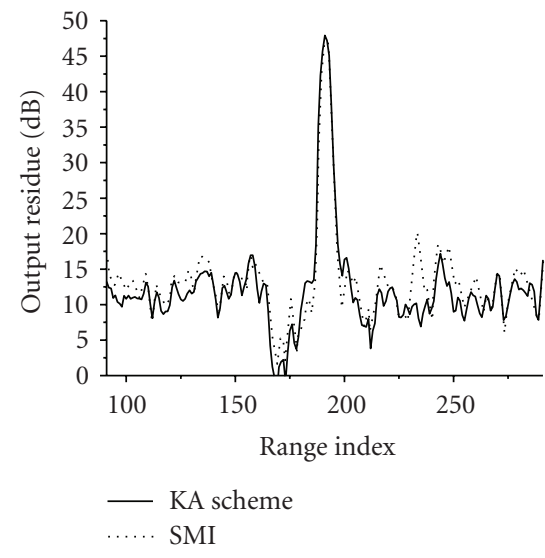

(d) target 4

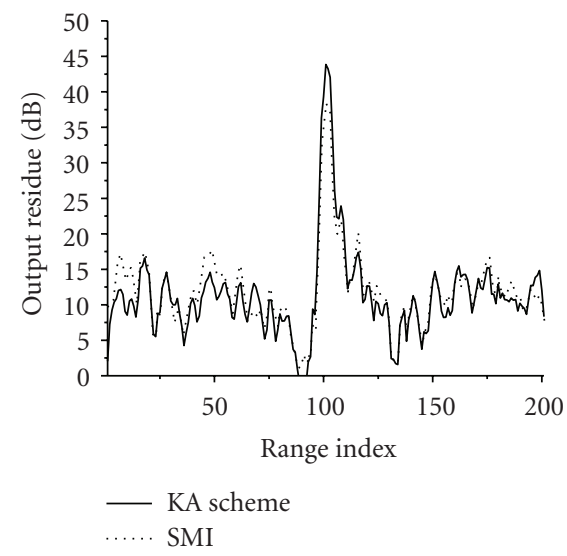

(e) target 5

Figure 7: Output residue (dB) versus range index for SMI and KA method. 24 samples are used for sample covariance matrix estimation.

or errors on the clutter signal whose characteristics may vary with angle of arrival, terrain, and so forth, $\mathbf{1}_{K \times 1}$ is a $K \times 1$ vector of ones, and $\mathbf{t}_{p}(\theta, \varphi)$ is a zero-mean random vector with typically tiny variance $[10,18]$.

We now consider the model of $C+N$ covariance matrix for multichannel SAR system, assuming that the clutter and noise vector are uncorrelated:

$$
\mathbf{R}=\mathbf{R}_{c}+\sigma_{n}^{2} \mathbf{I}_{K \times K}
$$

where $\mathbf{R}_{c}$ is the clutter covariance matrix, $\sigma_{n}^{2}$ is the noise power on each channel and pixel, and $\mathbf{I}_{K \times K}$ is a $K \times K$ identity matrix. Now consider $\mathbf{R}_{c}$ with the clutter vector denoted by the first term in (6):

$$
\begin{aligned}
\mathbf{R}_{c}= & E\left\{\left[\alpha_{c} \mathbf{c}(\theta, \varphi) \odot \boldsymbol{\varepsilon}(\theta, \varphi) \odot\left(\mathbf{1}_{K \times 1}+\mathbf{t}_{p}(\theta, \varphi)\right)\right]\right. \\
& \left.\times\left[\alpha_{c} \mathbf{c}(\theta, \varphi) \odot \boldsymbol{\varepsilon}(\theta, \varphi) \odot\left(\mathbf{1}_{K \times 1}+\mathbf{t}_{p}(\theta, \varphi)\right)\right]^{H}\right\} \\
= & \sigma_{c}^{2}\left[\mathbf{c}(\theta, \varphi) \mathbf{c}^{H}(\theta, \varphi)\right] \odot\left[\boldsymbol{\varepsilon}(\theta, \varphi) \boldsymbol{\varepsilon}^{H}(\theta, \varphi)\right] \\
& \odot\left[\mathbf{1}_{K \times K}+\mathbf{T}_{p}(\theta, \varphi)\right] .
\end{aligned}
$$

In (8), $\sigma_{c}^{2}=E\left[\alpha_{c} \alpha_{c}^{*}\right]$ denotes the clutter power concerning the discussing patch, $\mathbf{1}_{K \times K}$ represents the $K \times K$ matrix of ones, $\mathbf{T}_{p}(\theta, \varphi)=E\left[\mathbf{t}_{p}(\theta, \varphi) \mathbf{t}_{p}^{H}(\theta, \varphi)\right]$, and the results of covariance matrix taper (CMT) theory $[2,19]$ have been invoked. It is logical to recognize that the clutter covariance matrix is comprised of a deterministic component

$$
\sigma_{c}^{2}\left[\mathbf{c}(\theta, \varphi) \mathbf{c}^{H}(\theta, \varphi)\right] \odot\left[\boldsymbol{\varepsilon}(\theta, \varphi) \boldsymbol{\varepsilon}^{H}(\theta, \varphi)\right]
$$

and an unknown component

$$
\sigma_{c}^{2}\left[\mathbf{c}(\theta, \varphi) \mathbf{c}^{H}(\theta, \varphi)\right] \odot\left[\boldsymbol{\varepsilon}(\theta, \varphi) \boldsymbol{\varepsilon}^{H}(\theta, \varphi)\right] \odot \mathbf{T}_{p}(\theta, \varphi) .
$$

[10]. Thus, we can conclude from (7) and (8) that if the parameters, that is, $\sigma_{c}^{2}, \sigma_{n}^{2}, \mathbf{c}(\theta, \varphi)$, and $\boldsymbol{\varepsilon}(\theta, \varphi)$, at least the estimates of them, can be obtained a priori, it is not unreasonable to select

$$
\mathbf{R}_{0}=\sigma_{c}^{2}\left[\mathbf{c}(\theta, \varphi) \mathbf{c}^{H}(\theta, \varphi)\right] \odot\left[\boldsymbol{\varepsilon}(\theta, \varphi) \boldsymbol{\varepsilon}^{H}(\theta, \varphi)\right]+\sigma_{n}^{2} \mathbf{I}_{K \times K},
$$

as an initial guess of $\mathbf{R}$ to be incorporated in the estimation of $C+N$ covariance matrix. Hence we concern ourselves with the problem of finding the proper values of those parameters in the following discussion.

For a $K$-channel uniform linear array (ULA) with $d$ denoting the distance between each receiving phase center whose baseline lies along the flight direction, the spatial 


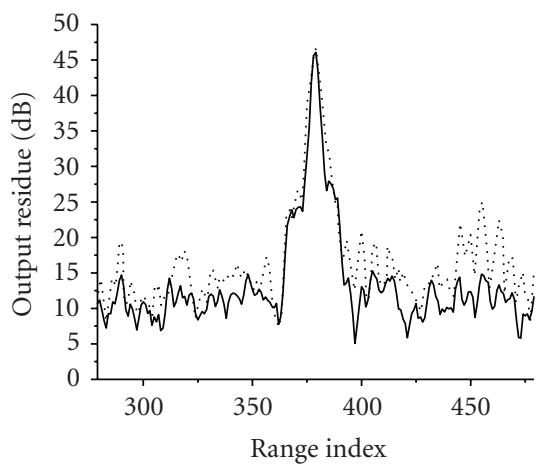

(a) target 1

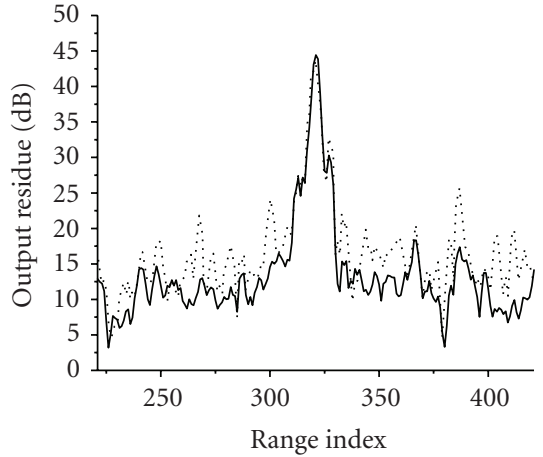

(b) target 2

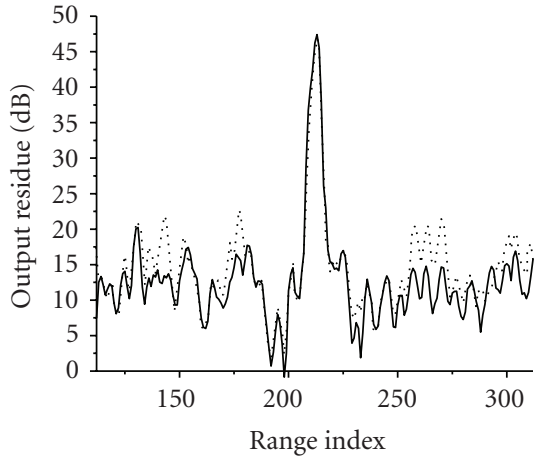

(c) target 3

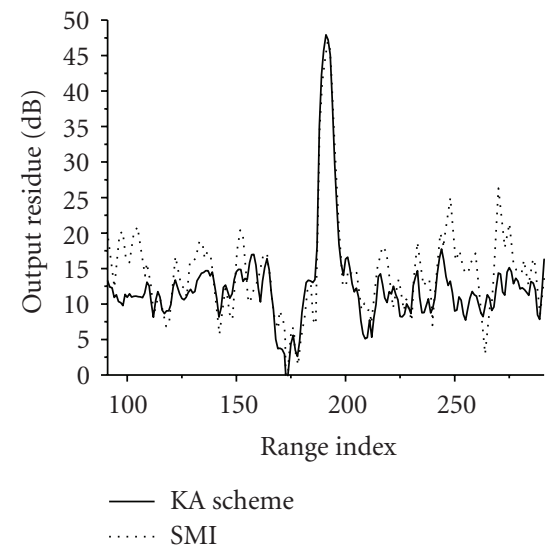

(d) target 4

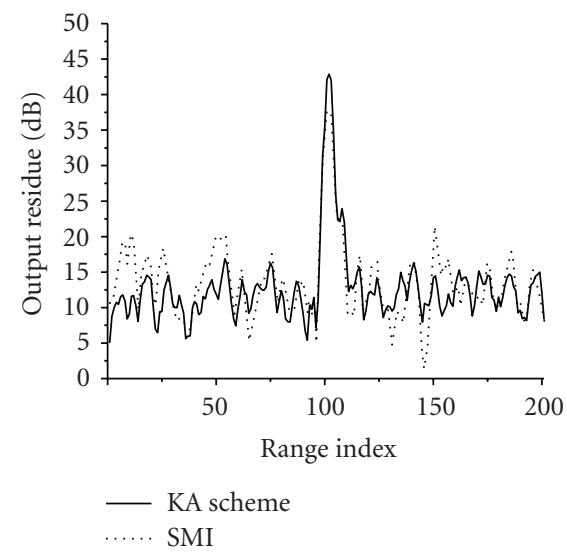

(e) target 5

FIgURE 8: Output residue (dB) versus range index for SMI and KA method. 12 samples are used for sample covariance matrix estimation.

steering vector, as a depiction of the ULA response to a point source with direction of arrival (DoA) $(\theta, \varphi)$, takes the form

$$
\mathbf{c}(\theta, \varphi)=\left[1, e^{j(2 \pi d / \lambda) \cos \theta \sin \varphi}, \ldots, e^{j(2 \pi d / \lambda)(K-1) \cos \theta \sin \varphi}\right]^{T}
$$

where $\lambda$ is the radar wavelength. It is obvious that the values of $d$ and $\lambda$ are totally known before flight, and the DOA for a certain clutter path is also available via reading the records of inertial navigation unit (INU). Thus we can obtain a relative accurate value of clutter steering vector if the values of these parameters are faithful.

$\boldsymbol{\varepsilon}(\theta, \varphi)$ denotes the mismatch between different receiving channels, whose value can be derived from the actual array manifold. However, in most cases, we can never know the perfectly accurate array manifold; several measures for exploiting the clutter signal itself to estimate $\varepsilon(\theta, \varphi)$ have been proposed $[18,20]$.

As for the noise power $\sigma_{n}^{2}$, we can use the designed signalto-noise ratio (SNR) of a receiver as an approximation or estimate it from the minimum eigenvalue in the eigenspectrum for a receiver test file. Besides, a simple estimation can be applied by averaging the clutter-free region in SAR image.

For estimating the clutter power $\sigma_{c}^{2}$, one can utilize knowledge source as digital terrain maps, land coverage data, or stationary scene SAR images as reference. Several works have been done for these estimations; for example, paper
[9] provides a method by employing digital maps, and [21] focuses the estimation on using SAR images.

Accomplishing the replacement of all the parameters with their obtained values yields the prior form of $\mathbf{R}$ to be used in the KA covariance matrix estimation as (5). However, the aforementioned value obtaining process for these parameters seems to be an inconvenient work in practical processing because (1) we may not have any digital maps or SAR images before flight; the terrain information can only be acquired by the $K$ SAR images formed in the flight; (2) the records of INU always present to be not accurate enough and lagging relative to the real values; (3) since spatial steering vector and the array errors vary with azimuth and elevation, we need to calculate them every time when process a new pixel under test. These problems motivate us to find a simple model of the prior $\mathrm{C}+\mathrm{N}$ covariance matrix. As we can see in the next part, by reasonable calibrating and compensating the multichannel SAR raw data, the model of a priori clutter covariance matrix is simplified to be a $K \times K$ matrix of ones with a scaling scalar tantamount to the local clutter power, based on which a KA adaptive SAR/GMTI algorithm is proposed for operating in real-world environments. part, a KA adaptive SAR/GMTI algorithm for multichannel 


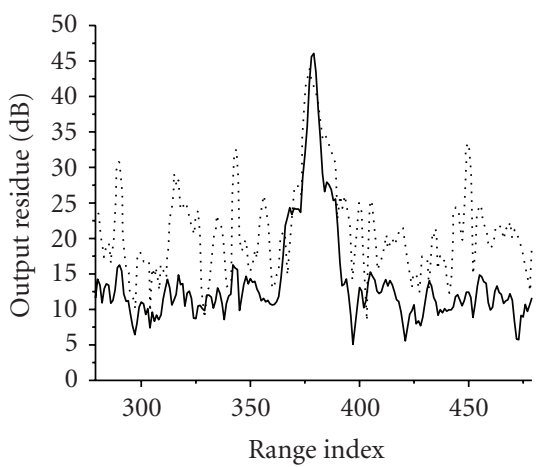

(a) target 1

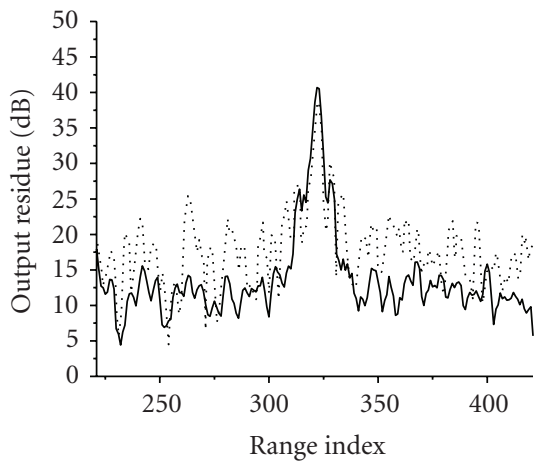

(b) target 2

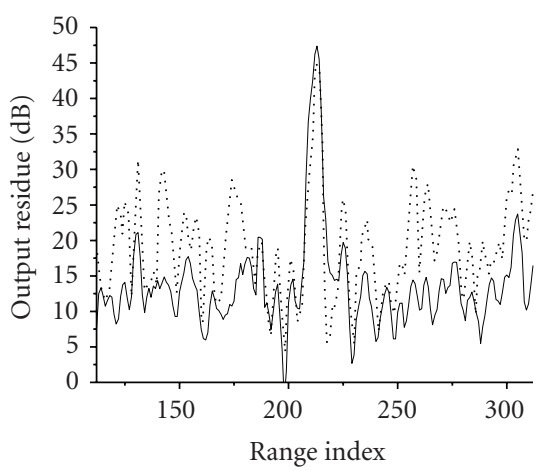

(c) target 3

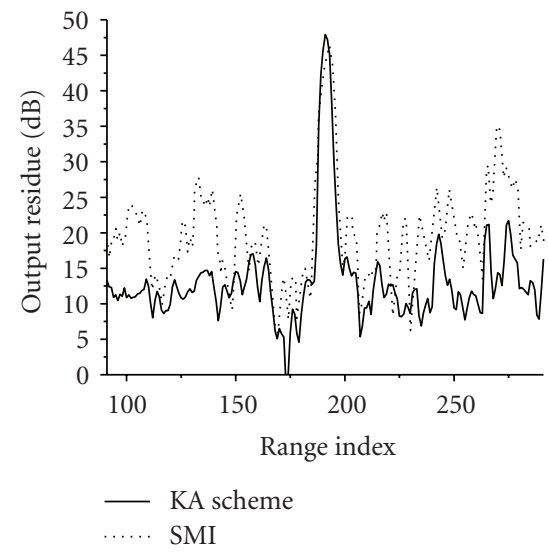

(d) target 4

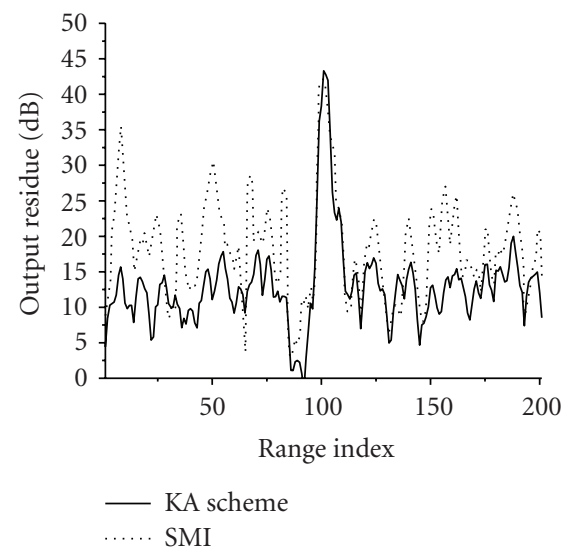

(e) target 5

FIGURE 9: Output residue $(\mathrm{dB})$ versus range index for SMI and KA method. 6 samples are used for sample covariance matrix estimation.

SAR system is proposed. It accomplishes GMTI processing by applying 6 major steps, which are identified by the shadowed blocks in the flow diagram of Figure 3. The acronym CM stands for covariance matrix.

The first step involves the calibration of array errors. The mismatch between different receiving channels is equalized by exploiting the received data itself. Several methods have been proposed for calibrating various kinds of errors in different domains; (see [22, 23]). In our experiment, we adopt the approach mentioned in [23] to calibrate the array errors in two-dimension frequency domain and the prior form of $C+N$ covariance matrix corresponding to (11) as follows:

$$
\begin{aligned}
\mathbf{R}_{0}^{\prime}= & \sigma_{c}^{2}\left[\mathbf{c}(\theta, \varphi) \mathbf{c}^{H}(\theta, \varphi)\right] \odot\left[\mathbf{1}_{K \times K}+\widetilde{\boldsymbol{\varepsilon}}(\theta, \varphi) \widetilde{\boldsymbol{\varepsilon}}^{H}(\theta, \varphi)\right] \\
& +\sigma_{n}^{2} \mathbf{I}_{K \times K},
\end{aligned}
$$

where $\widetilde{\boldsymbol{\varepsilon}}(\theta, \varphi)$ denotes the residual array error vector that can be sorted to the unknown component in (8); hence $\mathbf{R}_{0}^{\prime}$ can be rewritten as

$$
\mathbf{R}_{0}^{\prime}=\sigma_{c}^{2}\left[\mathbf{c}(\theta, \varphi) \mathbf{c}^{H}(\theta, \varphi)\right]+\sigma_{n}^{2} \mathbf{I}_{K \times K}
$$

As we know, since the phase lags induced by different along track positions of receiving antenna ports can be recognized as phase response mismatch between different receiving channels varying with DOA, they are also compensated in this step. Thus, the clutter steering vector for each pixel is reformatted to be a $K \times 1$ vector of ones, followed by the transformed $C+N$ covariance matrix which takes the form

$$
\mathbf{R}_{0}^{\prime}=\sigma_{c}^{2} \mathbf{1}_{K \times K}+\sigma_{n}^{2} \mathbf{I}_{K \times K} .
$$

Figure 4 provides the amplitude and phase responses of the three-channel SAR system before and after applying step 1 for comparison. All the response curves are normalized with respect to that of the center channel (channel 2). Therefore, a simplified form of the prior $C+N$ covariance matrix is generated for each pixel, which is determined completely by the clutter and noise power.

In step 2, the calibrated data for each channel undergo the image formation procedure and $K$ correlated maps are obtained for the same scenario.

Step 3 involves the construction of the prior $C+N$ covariance matrix for each pixel. As indicated by (15), this step can be accomplished by estimating the two parameters $\sigma_{c}^{2}$ and $\sigma_{n}^{2}$. The noise floor can be estimated by choosing the minimum eigenvalue of a sample covariance matrix or averaging over the clutter-free region in SAR images. Both 


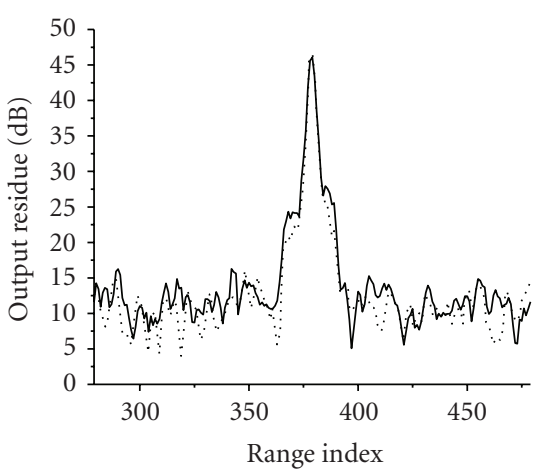

(a) target 1

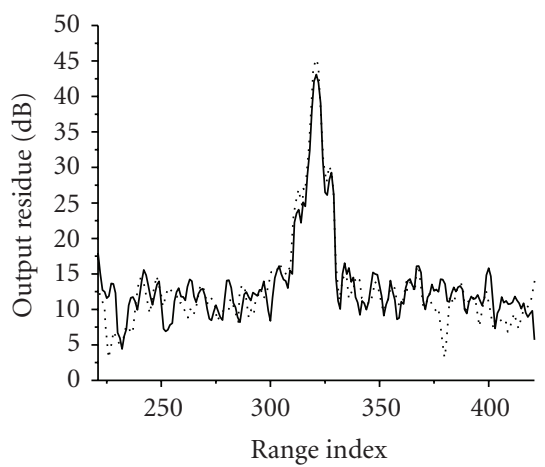

(b) target 2

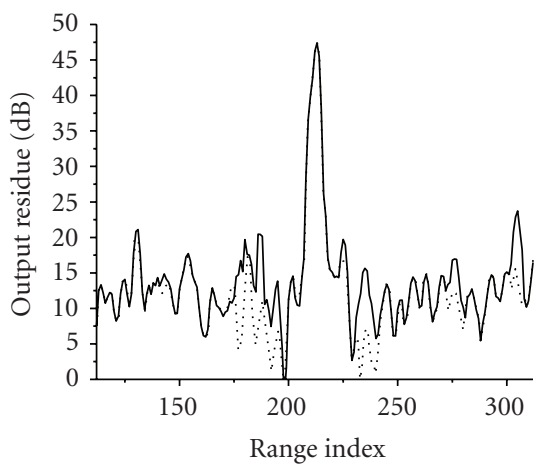

(c) target 3

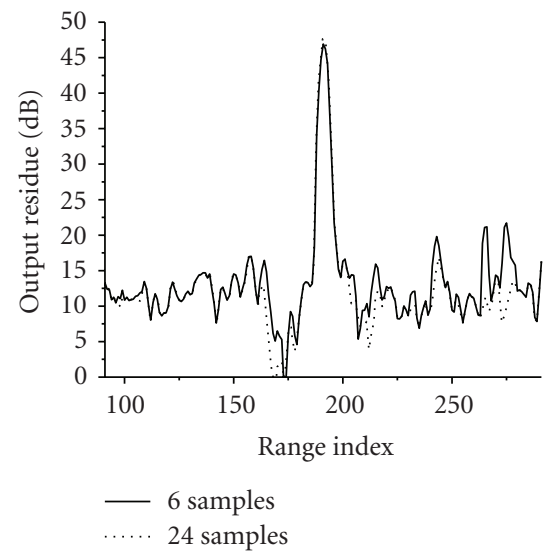

(d) target 4

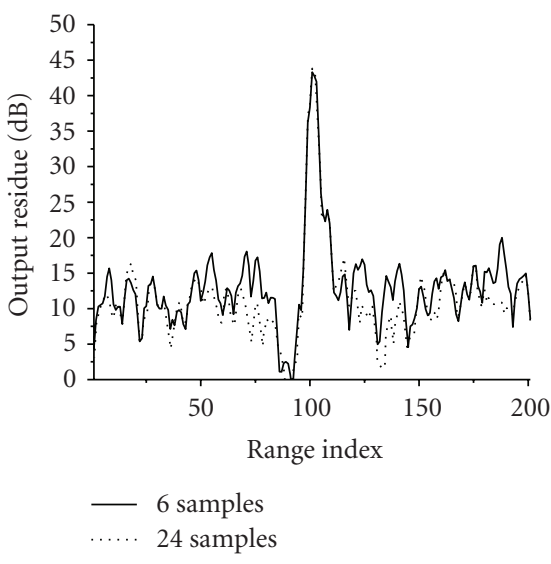

(e) target 5

Figure 10: Output residue (dB) versus range index for KA method using 6 and 24 samples, respectively.

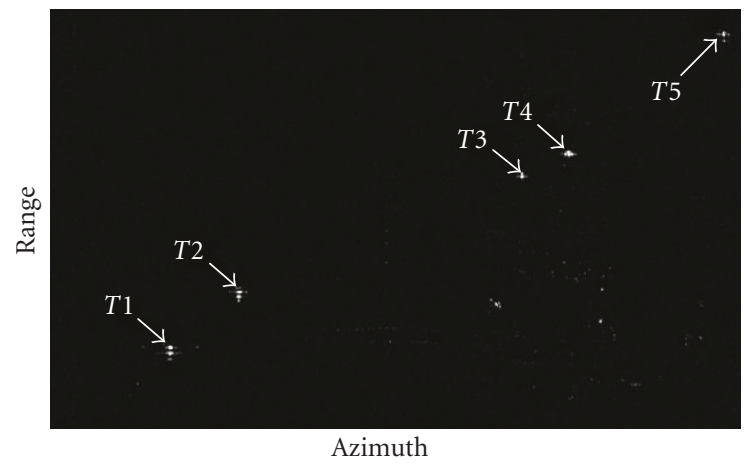

FIGURE 11: $\Sigma$ maps formed by KA scheme with 6 samples.

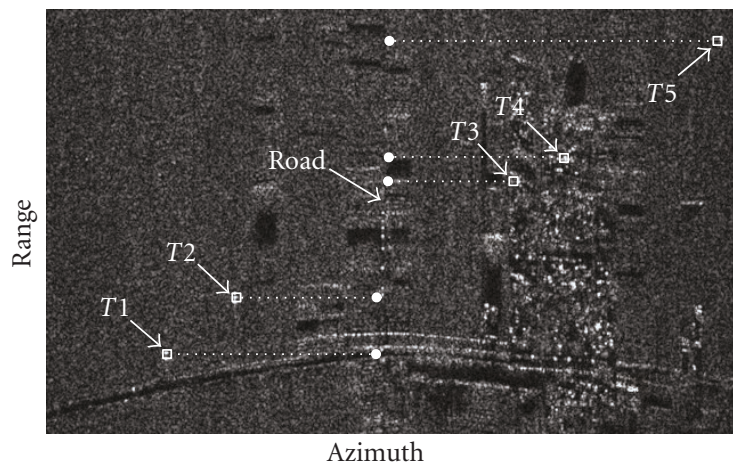

FIGURE 12: Reposition map of control targets. methods have been used for the three-channel SAR system and equivalent results are obtained. Since the receiver noise does not change over pixels, $\sigma_{n}^{2}$ is estimated only once and applied to the entire image.

We consider the situation that no a priori terrain information such as digital maps and locations of man-made features is known before flight; the only knowledge source is the multichannel SAR images. Given the data compensated in the aforementioned steps, we then wish to estimate the clutter voltage of by minimizing the mean-squared error
(MSE) as

$$
\min _{\alpha_{c}} E\left\{\left\|\mathbf{x}^{\prime}-\alpha_{c} \mathbf{1}_{K \times 1}\right\|^{2}\right\},
$$

where $\mathbf{x}^{\prime}$ denotes the input date after calibration and compensation in step 1 . One of the estimated solutions to (16) takes the form

$$
\hat{\alpha}_{c}=\frac{1}{L^{\prime} K} \sum_{i=1}^{L^{\prime}-1} \mathbf{x}^{\prime H}(i) \mathbf{1}_{K \times 1},
$$


followed by the estimation of clutter power calculated by $\hat{\sigma}_{c}^{2}=\left|\hat{\alpha}_{c}\right|^{2}$. In (17), $\mathbf{x}^{\prime}(i)$ denotes the samples vector after calibration in step 1 and $L^{\prime}$ the sample number for this estimation. In order to achieve an estimation of the local clutter power with high fidelity, the samples are drawn from the closest region adjacent to the pixel under test (containing the pixel under test) and $L^{\prime}$ is chosen to be small. Considering the present of targets in the sample set disturbing the estimation, the pixels which may comprise target signal should be removed. For doing this, a difference image between two images from different channels is formed, and the image pixels whose power exceeding a predetermined threshold (20 dB above the noise floor) are censored.

After step 1 3, a priori form of $C+N$ covariance matrix has been generated for each pixel. Step 4 is to concern the local training scheme, in which the sample covariance matrix $\widehat{\mathbf{R}}$ for each pixel is estimated using (2) with the sample vectors chosen from the adjacent range pixels (including several guard cells to avoid the cancellation of moving target itself) around the pixel under test.

Since the prior and sample forms of $C+N$ covariance matrix are available after accomplishing the former steps, a linear combination of them is generated using (5) in step 5. We use the method proposed in [12] to estimate the coefficient $\alpha$ as

$$
\hat{\alpha}=\frac{\rho}{\rho+\left\|\hat{\mathbf{R}}-\mathbf{R}_{0}\right\|^{2}},
$$

where $\rho=E\left\{\|\hat{\mathbf{R}}-\mathbf{R}\|^{2}\right\}$ and can be estimated by

$$
\hat{\rho}=\frac{1}{L^{2}} \sum_{i=1}^{L}\left\|\mathbf{x}^{\prime}(i)\right\|^{4}-\frac{1}{N}\|\hat{\mathbf{R}}\|^{2},
$$

where $\mathbf{x}^{\prime}(i)$ denotes the samples vector after calibration in step 1 . Hence the beamformer weight vectors for each pixel can be computed as

$$
\widetilde{\mathbf{w}}=\gamma \widetilde{\mathbf{R}}^{-1} \mathbf{s}
$$

followed by the formation of the modified $\Sigma$ map for target detection.

The last step contains CFAR processing, estimation of target's velocity, and target relocation. Many methods have been proposed for the estimation of moving target parameters, such as monopulse [24], adaptive monopulse [24], or maximum likelihood method [25]. Since this paper does not specifically address the problem of parameters estimation, detailed discussion is not provided herein. However, the experimental results of radial velocity estimation and reloaction of moving target using the maximum likelihood method are shown in the next section.

3.3. Improvement for Performance Loss. To give a comparison between the KA and local training SMI scheme, Figure 5 demonstrates the benefits of utilizing a priori knowledge for clutter suppression in multichannel SAR/GMTI processing by providing the SINR loss curves concerning the same pixel as in Figure 2 for ideal, sample and linear combination $C+N$ covariance matrix, respectively. As we can see, by introducing the KA estimation of $\mathbf{R}$, the SINR loss curve has approached the ideal one, which is more clear for the situation of less sample support, see (a), (b). When 24 samples used, excellent coincidence can be seen between the KA and ideal curve, see (c). It can be seen that, the gap between the two methods is diminished with increasing samples which indicates that the SMI method using large number of samples may provide satisfying performance. We caution, however, adding more samples would typically degrade the sensitivity in detection targets with low radial velocities and might actually increase the chances of target-like signal falling into the samples set $[6,10]$.

\section{Experimental Results}

In this section, experimental clutter mitigation results with respect to KA algorithm and traditional SMI method are presented for comparison. The data described were also collected by the three-channel SAR system with the principal parameters listed in Table 1 and a ground area including 5 control targets (cars with different radial velocities) was imaged. Since this experiment focused on the performance of slowly moving target indication, the PRF of $1250 \mathrm{~Hz}$ is enough to avoid the alias of moving target in Doppler. Figure 6 provides the SAR image of the discussed scene from the central receiving channel. To identify the precise position of the road where 5 control targets were moving, several corner reflectors had been arranged along the road before flight, which can be found in the central part of the SAR image. 5 control targets $(T 1 \sim T 5)$ with varied radial velocities show different azimuth displacements in the image, which are marked by the white arrows.

With SAR image generated from each receiving channel, the KA and local training SMI algorithm were applied for clutter suppression, respectively. To achieve a fair comparison, the data were also preprocessed using Ender's 2D calibration algorithm [23] with 1024 receiving pulses before SMI processing. We now examine how the algorithms performed against the real-world clutter environment. We first present the data as plots of output power residue (after adaptive processing) around the 5 control targets versus the range index. In each plot, a control target is at the central range gate and its return essentially extends to about 1015 right and left adjacent gates. Thus the data from the pixel under test and its adjacent range pixels (guard cells) are not used for $C+N$ covariance matrix estimation when we adaptively process the data. The $L$ sample vectors are formed from the $L / 2$ data vectors associated with the range cells to the immediate right and left of the right and left guard cells, respectively. We evaluate and plot the output residue in windows ( \pm 100 range pixels) around the range pixels occupied by the 5 control targets.

In Figures 7-9, the output residue around control targets versus the range gate index is plotted for the sample number 
TABLE 2: Results of radial velocity estimation and relocation concerning the control targets.

\begin{tabular}{lcccc}
\hline $\begin{array}{l}\text { Target } \\
\text { number }\end{array}$ & \multicolumn{2}{c}{$\begin{array}{c}\text { Radial velocity } \\
(\mathrm{m} / \mathrm{s})\end{array}$} & \multicolumn{2}{c}{$\begin{array}{c}\text { Azimuth position in SAR image } \\
\text { (pixel) }\end{array}$} \\
& Real value & Estimation & Real value & Estimation \\
\hline T1 & -4.16 & -3.95 & 516 & 512 \\
$T 2$ & -2.77 & -2.59 & 516 & 513 \\
$T 3$ & +2.51 & +2.38 & 516 & 518 \\
$T 4$ & +3.34 & +3.17 & 516 & 519 \\
T5 & +5.98 & +5.88 & 517 & 519 \\
\hline
\end{tabular}

$L$, equal to $24,12,6$, respectively. It is seen from Figure 7 ( $L=24$ ) that the performance of KA method seems to have slight improvement relative to the SMI method, except that the output power of the 5th target with respect to KA method is about $5 \mathrm{~dB}$ better than the value with respect to SMI. For this relatively large number of samples, all the control targets are about $35 \mathrm{~dB}$ above the clutter power residue. For $L=$ 12 (Figure 8), the performance of KA method is noticeably better than the SMI method in terms of the target peak to average power level outside the target gates. And for $L=6$ (Figure 9) there is even significant improvement of proposed KA algorithm relative to the traditional SMI method, which is on the order of $10 \sim 15 \mathrm{~dB}$. For this relatively small number of samples, the performance of KA method still seems to be graceful while the performance of local training SMI method degrades to an intolerable level that is totally unqualified for the following CFAR test.

After the comparison between clutter suppression performances associated with the different algorithms, we now focus on the convergence of the proposed algorithm in heterogeneous environments. In Figure 10, we plot the output residue around the 5 control targets associated with the KA algorithm for $L$, equal to 24, 6, respectively. As can be seen, there is no significant performance loss when training samples decrease from 24 to 6. Acceptable clutter suppression results are achieved with less sample support for the KA algorithm, which indicated its fast convergence relative to that of SMI method.

With the beamforming applied, Figure 11 shows the modified $\Sigma$ map formed by the KA method with 6 training samples, in which we can have an overview of the clutter mitigation performance. This map then undergoes a CACFAR processing and all the control targets are detected with the false alarm rate equal to $10^{-6}$.

Finally, the radial velocity estimation and relocation of the detected targets is implemented. Figure 12 describes the relocation result of the control targets in SAR image, in which the estimated positions are marked by the white dots. As we can see, all the targets are relocated to the azimuth positions approximately on the road. In Table 2 , the detailed results of relocation as well as velocity estimation are listed, where an azimuth pixel is equal to $4.2 \mathrm{~m}$ and the radial velocity with "-" or "+" denotes the toward or backward motion of the control targets relative to the radar, respectively.

\section{Conclusions}

An effective method was developed for clutter suppression of a multichannel SAR/GMTI system performs in heterogeneous environment in this paper. It arose from the knowledge-aided STAP schemes and named as KA adaptive SAR/GMTI algorithm. In this algorithm, the prior form of $C+N$ covariance matrix concerning each image pixel is reformed to be a $K \times K$ matrix of ones multiplied by the local clutter power plus a $K \times K$ identity matrix multiplied by the thermal noise power. The prior form is incorporated in the estimating of the $C+N$ covariance matrix to achieve an accurate estimate relative to the local training scheme. And then, clutter suppression beamforming is applied with the weight vector calculated by the KA $C+$ $N$ covariance matrix. This new algorithm reduces sample support by its fast convergence properties and results in a clutter suppression performance that is more robust to nonstationary clutter distribution relative to the traditional scheme, which has been proved by the experimental clutter suppression results from a three-channel SAR system. These virtues make the algorithm to be an advisable choice for ground slowly moving targets detection in real-world clutter environments.

\section{Acknowledgments}

This work was supported by a Grant from the Aeronautical Science Foundation (no. 20080152004) and the Ph.D. Programs Foundation of Ministry of Education of China (no. 20070280531).

\section{References}

[1] J. Ward, "Space-time adaptive processing for airborne radar," Tech. Rep. ESC-94-109, Lincoln Laboratory, 1994.

[2] J. R. Guerci, Space-Time Adaptive Processing for Radar, Artech House, Boston, Mass, USA, 2003.

[3] R. Klemm, Principles of Space-Time Adaptive Processing, IEE Press, London, UK, 2002.

[4] E. Yadin, "Evaluation of noise and clutter induced relocation errors in SAR GMTI," in Proceedings of the IEEE International Radar Conference (RADAR '95), pp. 650-655, Alexandria, Va, USA, May 1995.

[5] E. Yadin, "A performance evaluation model for a two port interferometer SAR-MTI," in Proceedings of the IEEE National Radar Conference, pp. 261-266, Ann Arbor, Mich, USA, May 1996.

[6] W. L. Melvin, "Space-time adaptive radar performance in heterogeneous clutter," IEEE Transactions on Aerospace and Electronic Systems, vol. 36, no. 2, pp. 621-633, 2000.

[7] W. L. Melvin and J. R. Guerci, "Adaptive detection in dense target environments," in Proceedings of the IEEE Radar Conference, pp. 187-192, Atlanta, Ga, USA, May 2001.

[8] J. S. Bergin, P. M. Techaui, W. L. Melvin, and J. R. Guerci, "GMTI STAP in target-rich environments: site-specific analysis," in Proceedings of the IEEE Radar Conference, pp. 22-25, Long Beach, Calif, USA, April 2002.

[9] J. R. Guerci and E. J. Baranoski, "Knowledge-aided adaptive radar at DARPA: an overview," IEEE Signal Processing Magazine, vol. 23, no. 1, pp. 41-50, 2006. 
[10] J. S. Bergin, C. M. Teixeira, P. M. Techau, and J. R. Guerci, "Improved clutter mitigation performance using knowledgeaided space-time adaptive processing," IEEE Transactions on Aerospace and Electronic Systems, vol. 42, no. 3, pp. 997-1009, 2006.

[11] X. Zhu, J. Li, and P. Stoica, "Knowledge-aided adaptive beamforming," IET Signal Processing, vol. 2, no. 4, pp. 335345,2008

[12] P. Stoica, J. Li, X. Zhu, and J. R. Guerci, "On sing a priori knowledge in space-time adaptive processing," IEEE Transaction on Signal Processing, vol. 56, no. 6, pp. 2598-2602, 2008.

[13] I. G. Cumming and F. H. Wong, Digital Processing of Synthetic Aperture Radar Data: Algorithms and Implementation, Artech House, Boston, Mass, USA, 2005.

[14] D. Zhu, S. Ye, and Z. Zhu, "Polar format agorithm using chirp scaling for spotlight SAR image formation," IEEE Transactions on Aerospace and Electronic Systems, vol. 44, no. 4, pp. 1433$1448,2008$.

[15] D. Zhu, X. Mao, Y. Li, and Z. Zhu, "Far-field limit of PFA for moving target imaging," to appear in IEEE Transactions on Aerospace and Electronic Systems.

[16] I. S. Reed, J. D. Mallet, and L. E. Brennan, "Rapid convergence rate in adaptive arrays," IEEE Transactions on Aerospace and Electronic Systems, vol. 10, no. 6, pp. 853-863, 1974.

[17] T. W. Anderson, An Introduction to Multivariate Statistical Analysis, John Wiley \& Sons, New York, NY, USA, 2nd edition, 1984.

[18] W. L. Melvin and G. A. Showman, "An approach to knowledge-aided covariance estimation," IEEE Transactions on Aerospace and Electronic Systems, vol. 42, no. 3, pp. 1021-1041, 2006.

[19] J. R. Guerci, "Theory and application of covariance matrix tapers for robust adaptive beamforming," IEEE Transactions on Signal Processing, vol. 47, no. 4, pp. 985-997, 1999.

[20] W. Wirth, Radar Techniques Using Array Antennas, The Institution of Electrical Engineers, London, UK, 2001.

[21] N. Goodman and P. R. Gurram, "STAP training through knowledge-aided predictive modeling," in Proceedings of the IEEE Radar Conference, pp. 388-393, Philadelphia, Pa, USA, April 2004.

[22] M. Soumekh, "Signal subspace fusion of uncalibrated sensors with application in SAR and diagnostic medicine," IEEE Transactions on Image Processing, vol. 8, no. 1, pp. 127-137, 1999.

[23] J. H. G. Ender, “The airborne experimental multi-channel SAR-system AER-II," in Proceedings of the European Conference on Synthetic Aperture Radar (EUSAR '96), pp. 49-52, Königswinter, Germany, May 1996.

[24] U. Nickel, "Overview of generalized monopulse estimation," IEEE Aerospace and Electronic Systems Magazine, vol. 21, no. 6, pp. 27-56, 2006.

[25] J. H. G. Ender, "Detection and estimation of moving target signals by multi-channel SAR," in Proceedings of the European Conference on Synthetic Aperture Radar (EUSAR '96), pp. 411417, Königswinter, Germany, May 1996. 